\title{
PAPER
}

\section{Bilateral hemispheric alteration of memory processes in right medial temporal lobe epilepsy}

\author{
S Dupont, Y Samson, P-F Van de Moortele, S Samson, J-B Poline, D Hasboun, \\ D Le Bihan, M Baulac
}

J Neurol Neurosurg Psychiatry 2002;73:478-485

See end of article for authors' affiliations

.....................

Correspondence to: Dr S Dupont, CEA-SHFJ, 4 place du Général Leclerc, 91406 Orsay, France; dupont@shfi.cea.fr

Received 27 July 2001 In revised form 23 November 2001 Accepted 6 June 2002

\begin{abstract}
Objective: Functional MRI (fMRI) was used to investigate right medial temporal lobe epilepsy (RTLE) effects on verbal memory.

Methods: BOLD FMRI data were collected from seven right sided MTLE patients (RTLE) and compared with the data previously acquired from seven left sided MTLE patients (LTLE) and 10 control subjects. Twenty two contiguous images covering the whole brain were acquired using an EPI echoplanar sequence. Subjects were instructed to learn a list of 17 words, and to recall it immediately and at 24 hours interval. Group analyses were performed using SPM96.

Results: RTLE patients retrieval performances were significantly impaired as compared with the performance of control subjects. As compared with control subjects and LTLE patients, RTLE patients exhibited a different pattern of hemispheric activations and a global decrease in left hemisphere functional activity.

Conclusion: MTLE cannot be considered as a model of pure well lateralised hippocampal dysfunction. The verbal memory impairment depicted in RTLE patients may be considered as the witness of a bilateral impairment of the neuroanatomical circuits subserving memory.
\end{abstract}

M emory impairment is a dominant complaint in medial temporal lobe epilepsy (MTLE). A large number of epilepsy related factors may influence memory functions in MTLE patients: the presence and the lateralisation of the medial temporal lobe structural lesions, such as hippocampal sclerosis, ${ }^{1}$ the epileptic process (age at onset of the epileptic seizures, duration of epilepsy, seizure frequency, seizure activity, and propagation $)^{2}$ and of course, the cognitive side effects of antiepileptic medication. ${ }^{3}$ It seems probable that all these factors contribute to the memory deficit, which must be regarded as a multifactorial condition. The relative contribution of each separate factor is difficult to establish but numerous studies have shown that the side of the lesion and of the seizure focus was a determinant factor. In MTLE that is supposed to be a model of "pure well-lateralised hippocampal dysfunction", verbal memory deficit is often associated with left sided MTLE (LTLE) whereas visuo-spatial memory deficit is associated with right sided MTLE (RTLE). ${ }^{4}$ However, electroclinical, neuroradiological, and pathological evidences suggest that bilateral hippocampal involvement, whether obvious or subtle, is present in some degree in most MTLE patients and provide supports for bilateral consequences of the MTLE syndrome. ${ }^{5}$ In a previous paper, ${ }^{6}$ we used functional MRI (fMRI) to compare the neuroanatomical circuits subserving episodic memory in LTLE patients and control subjects. We demonstrated that LTLE patients activated an abnormal neocortical and mediotemporal network with a new aberrant activation of the left dorsolateral prefrontal cortex, the disappearance of the right hippocampal activation, and the decrease of the bilateral parahippocampal activations, especially during the retrieval tasks. The disappearance of the right hippocampal and parahippocampal activation led us to suggest that the left hippocampal dysfunction, whether linked to the epilepsy or to the structural lesion-that is, the hippocampal sclerosis-could lead to a right hippocampal dysfunction, providing evidence for the bilateral functional consequences of the MTLE syndrome.

This first finding led us to question whether, conversely, a right hippocampus dysfunction could lead to a left hippocam- pal dysfunction. Therefore, we used functional magentic resonance imaging (fMRI) to investigate in RTLE patients the brain networks that were activated during a verbal episodic memory task known to activate mostly the left hemisphere in both control subjects and LTLE patients. We sought to determine if RTLE patients had bilateral hemispheric dysfunction.

\section{METHODS}

\section{Patients and controls}

The study population included seven patients (three women and four men; mean age: 34 years; range: 18-53) with right MTLE who were undergoing presurgical evaluation for anterior temporal lobectomy at the Pitié-Salpêtrière Epilepsy Unit. They were matched with a control group, consisting of seven patients with left MTLE (five women and two men, mean age: 38 years, range: $30-54)$, and 10 healthy volunteers (eight women and two men; mean age: 25.4 ). All subjects gave informed written consent in accordance with the Declaration of Helsinki and the local ethics committee approved the study. The seven right MTLE (RTLE) patients underwent the same presurgical evaluation ${ }^{7}$ as the left MTLE (LTLE) patients, including: medical, neurological, and neuropsychological examinations, video-EEG monitoring, brain MRI, FDG-PET examinations. The inclusion criteria for our study were also the same for both RTLE and LTLE patients: right handed lateralisation; surface EEG recordings consistent with right sided medial temporal lobe seizure onset; right hippocampal sclerosis diagnosed on MRI without any other structural abnormalities.

In a second step, all the patients achieved the presurgical evaluation and underwent an anterior temporal lobectomy.

Abbreviations: $\mathrm{FMRI}$, functional magnetic resonance imaging; MTLE, medial temporal lobe epilepsy; RTLE, right sided medial temporal lobe epilepsy; LTLE, left sided medial temporal lobe epilepsy 
The RTLE patients all belong to Engel's class I with a postoperative outcome exceeding one year. ${ }^{8}$ LTLE patients respectively belong to Engel's class I $(n=5)$ and III $(n=1)$ with a postoperative outcome exceeding three years, one patient finally refused to undergo surgery.

\section{RTLE patients data}

During their presurgical evaluation, all patients underwent surface video-EEG and one patient underwent an intracranial EEG (SEEG). Ictal scalp recordings demonstrated right unilateral temporal lobe onset in six patients and bilateral temporal lobe seizure onset in one patient who further underwent SEEG. On scalp video-EEG, bilateral medial temporal interictal spikes were present in one patient (the SEEG patient), the other patients had only right temporal interictal spikes or no interictal spikes at all. In the patient who underwent SEEG, ictal intracranial recordings demonstrated right unilateral temporal lobe onset for all the seizures that were recorded.

The seven RTLE patients were taking antiepileptic drugs. The number and the doses of the antiepileptic drugs were relatively equivalent across the patients and were not withrawn at the time of fMRI.

\section{fMRI procedures}

Imaging parameters and acquisition procedures were identical to the methods described in a previous paper. ${ }^{6}$ fMRI technique is based on the BOLD (blood oxygenation level dependent) effects-that is, the susceptibility effects produced by changes in deoxyhaemoglobin concentration such that focal alterations in cerebral haemodynamics can be visualised. ${ }^{9}$ BOLD fMRI data were acquired on a 3-Tesla Bruker system equipped with a prototype fast gradient system and the standard quadrature head coil. Subjects were placed in a supine position in the MRI scanner. Their heads were immobilised with cushions to reduce motion artefact. The stimuli were projected onto a mirror located at the end of the scanner bore. Subjects were equipped with prism glasses that allowed them to see the projection in central vision without image distortion. For each subject, conventional structural sagittal and axial inversion recovery turbo flash $\mathrm{Tl}$ weighted localiser images were first collected to provide detailed anatomic information. An automatic shimming method further enhanced the signal to noise ratio and reduced the fMRI susceptibility artefacts, especially within the temporal lobe. After the acquisition of these anatomical images, gradient echo echoplanar (EPI) fMRI was performed in 22 contiguous $5 \mathrm{~mm}$ axial slices (repetition time $=6$ seconds, $64 \times 80$ matrix, $22 \mathrm{~cm}^{2}$ field of view) covering the whole brain.

EPI sequences are sensitive to signal changes arising from small changes in blood oxygenation levels and permit a complete brain coverage in a short period of time.

The entire session, including both structural and functional sequences lasted 45 minutes the first day and 50 minutes the following day.

\section{Memory task procedures}

Memory tasks included verbal episodic memory encoding and retrieval tasks. The aim of the experiment was to create two retrieval conditions that differed in relation to immediate retrieval or 24 hours delayed retrieval. Two sessions were performed on consecutive days. A sequential task-activation paradigm was used, alternating between an experimental condition and a baseline condition (see fig 1). The baseline condition was the same for all the experiments and consisted of the fixation of the letter A. Scanning was performed over a 324 second block. Each block included five control and four experimental conditions beginning with the control condition.

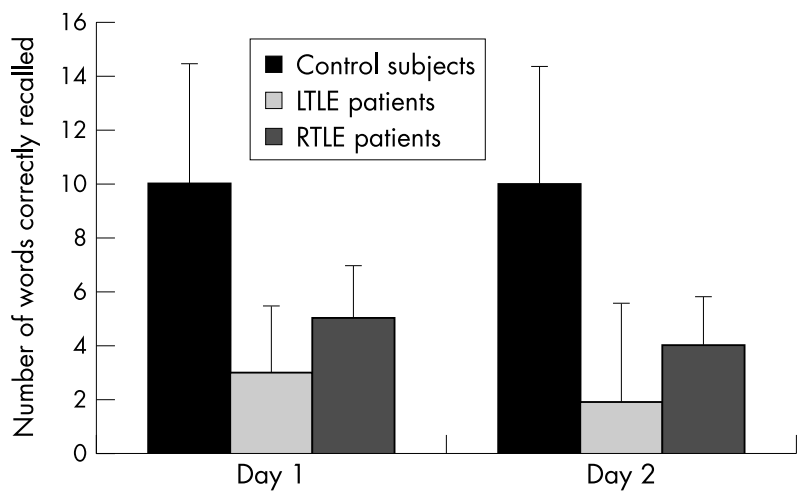

Figure 1 Memory test performances: comparison between the first day and the second day.

Session 1 (day 0)

As previously described, ${ }^{6}$ two memory experimental blocks were performed on the first day. The encoding experimental block consisted in learning a supra-span list of 17 abstract words that has been used to demonstrate verbal episodic memory deficits in epileptic patients. ${ }^{10}$ In the same block, the list of words was presented four times, alternating with baseline condition. The words were displayed at the rate of one word every two seconds. Subjects were instructed to learn the words silently for later recall. At the end of the encoding block, subjects rested for two minutes. During the following retrieval experimental condition (immediate retrieval), subjects were asked to recall silently the words that they had previously learned. At the end of the session, subjects were tested to establish the efficiency of the retrieval of the verbal information and were asked to recall out loud the words that they had previously learned.

Session 2 (day 1)

The following day, subjects were visually instructed to recall silently the list of words that they had learned the previous day (delayed retrieval). At the end of the session, subjects were again tested to establish the efficiency of the delayed retrieval and were asked to recall loudly the words that they had previously learned.

\section{Image analysis}

fMRI data were analysed on SPARC workstations (Sun Microsystems, Mountain View, CA). Statistical analysis was performed in MATLAB (Mathworks, Natick, Mass, USA) using a statistical parametric mapping (SPM) software package (SPM 96). ${ }^{11}$

\section{Statistical analysis}

For each subject, we performed a stereotaxic reorientation of the images along the bi-commissural line. Images were then coregistered and resliced to correct movement and further spatially transformed to standard stereotaxic coordinates to correct for anatomical variance across subjects. The standard reference space used in SPM96 is based on the MNI reference space. The resulting images were convolved with a three dimensional Gaussian filter to suppress noise. The data were then analysed statistically on a voxel by voxel basis using a two temporal basis functions model. We first performed an individual analysis for each subject and then a multisubject analysis using a similar method. As our aim was to investigate the global changes in neocortical activations in relation to the hippocampal damage, we focused our study on the group analysis. For this multisubject analysis, a given voxel was considered to be significantly activated if, on comparison with a reference task, there was an increase in the haemodynamic response function at $\mathrm{p}<0.0001(\mathrm{p}<0.01$ corrected for multiple 
Table 1 Activation foci in normal control subjects), left and right epileptic patients during the encoding tests (stereotaxic coordinates are expressed in $\mathrm{mm}$ )

\begin{tabular}{|c|c|c|c|c|c|c|c|c|c|c|c|c|c|c|c|c|c|c|c|c|c|c|c|c|}
\hline \multirow[b]{3}{*}{ Region } & \multicolumn{12}{|c|}{ Left hemisphere } & \multicolumn{12}{|c|}{ Right hemisphere } \\
\hline & \multicolumn{4}{|c|}{ CTRL } & \multicolumn{4}{|l|}{ LTLE } & \multicolumn{4}{|l|}{ RTLE } & \multicolumn{4}{|c|}{ CTRL } & \multicolumn{4}{|c|}{ LTLE } & \multicolumn{4}{|c|}{ RTLE } \\
\hline & $x$ & $y$ & z & $\begin{array}{l}\text { z } \\
\text { value }\end{array}$ & $\mathrm{x}$ & $y$ & $\mathrm{z}$ & $\begin{array}{l}z \\
\text { value }\end{array}$ & $\mathrm{x}$ & y & z & $\begin{array}{l}\text { z } \\
\text { value }\end{array}$ & $x$ & y & z & $\begin{array}{l}\mathrm{z} \\
\text { value }\end{array}$ & $\mathrm{x}$ & y & $z$ & $\begin{array}{l}\text { z } \\
\text { value }\end{array}$ & $x$ & y & z & $\begin{array}{l}z \\
\text { value }\end{array}$ \\
\hline \multicolumn{25}{|l|}{ Post Cx } \\
\hline $\mathrm{OCx}$ & $\begin{array}{l}-15 \\
-18 \\
-27\end{array}$ & $\begin{array}{l}-72 \\
-81 \\
-75\end{array}$ & $\begin{array}{l}12 \\
15 \\
21\end{array}$ & $\begin{array}{l}4.5 \\
5.7 \\
7.3\end{array}$ & & & & & & & & & $\begin{array}{l}15 \\
18 \\
21\end{array}$ & $\begin{array}{l}-66 \\
-75 \\
-84\end{array}$ & $\begin{array}{l}12 \\
12 \\
15\end{array}$ & $\begin{array}{l}5.7 \\
4.3 \\
8.1\end{array}$ & & & & & & & & \\
\hline $\begin{array}{l}\text { OT Cx } \\
37 / 41 / \\
42\end{array}$ & & & & & & & & & & & & & & & & & & & & & & & & \\
\hline$P C x$ & -33 & -51 & 48 & 6.5 & -33 & -48 & 48 & 5.01 & & & & & & & & & 36 & -42 & 48 & 4.63 & 33 & -45 & 45 & 5.91 \\
\hline $40 / 7$ & $\begin{array}{l}-33 \\
-30\end{array}$ & $\begin{array}{l}-60 \\
-66\end{array}$ & $\begin{array}{l}45 \\
39\end{array}$ & $\begin{array}{l}6.5 \\
5.2\end{array}$ & -45 & -33 & 39 & 5.77 & & & & & & & & & $\begin{array}{l}30 \\
33\end{array}$ & $\begin{array}{l}-51 \\
-54\end{array}$ & $\begin{array}{l}57 \\
48\end{array}$ & $\begin{array}{l}4.71 \\
5.07\end{array}$ & 39 & -45 & 54 & 4.03 \\
\hline $\begin{array}{l}T C x \\
\text { ST Cx } \\
22\end{array}$ & -57 & -36 & 6 & 5.6 & $\begin{array}{l}-63 \\
-54\end{array}$ & $\begin{array}{l}-36 \\
-42\end{array}$ & $\begin{array}{l}3 \\
3\end{array}$ & $\begin{array}{l}5.04 \\
4.99\end{array}$ & & & & & & & & & & & & & & & & \\
\hline $\begin{array}{l}F C x \\
\text { Ventro } \\
\text { lateral } C x \\
44 / 45\end{array}$ & $\begin{array}{l}-54 \\
-39\end{array}$ & $\begin{array}{l}24 \\
27\end{array}$ & $\begin{array}{l}6 \\
3\end{array}$ & $\begin{array}{l}6.8 \\
4.4\end{array}$ & $\begin{array}{l}-54 \\
-36 \\
-36\end{array}$ & $\begin{array}{l}27 \\
24 \\
48\end{array}$ & $\begin{array}{l}9 \\
9 \\
12\end{array}$ & $\begin{array}{l}5.85 \\
6.22 \\
4.15\end{array}$ & & & & & 42 & 27 & 9 & 7.1 & & & & & & & & \\
\hline $\begin{array}{l}\text { Dorso } \\
\text { lateral Cx } \\
6 / 9 / 10 / \\
46\end{array}$ & & & & & $\begin{array}{l}-57 \\
-57 \\
-51 \\
-42 \\
-42 \\
-48 \\
-36 \\
-33\end{array}$ & $\begin{array}{l}0 \\
27 \\
30 \\
48 \\
54 \\
21 \\
42 \\
60\end{array}$ & $\begin{array}{l}33 \\
24 \\
30 \\
\\
24 \\
18 \\
30 \\
21 \\
12\end{array}$ & $\begin{array}{l}4.28 \\
5.46 \\
7.22 \\
\\
5.1 \\
4.18 \\
7.05 \\
3.92 \\
4.47\end{array}$ & $\begin{array}{l}-57 \\
-54 \\
-45\end{array}$ & $\begin{array}{l}6 \\
-6 \\
6\end{array}$ & $\begin{array}{l}39 \\
45 \\
36\end{array}$ & $\begin{array}{l}4.32 \\
4.74 \\
7.21\end{array}$ & & & & & $\begin{array}{l}42 \\
27 \\
33 \\
\\
36\end{array}$ & $\begin{array}{l}30 \\
57 \\
51 \\
45\end{array}$ & $\begin{array}{l}30 \\
27 \\
30 \\
21\end{array}$ & $\begin{array}{l}6.9 \\
4.95 \\
5.43 \\
4.99\end{array}$ & & & & \\
\hline $\begin{array}{l}\text { Med Cx } \\
\text { CG Cx } \\
23 / 30 / \\
31\end{array}$ & & & & & & & & & & & & & & & & & & & & & & & & \\
\hline $\begin{array}{l}\text { CG Cx } \\
32\end{array}$ & & & & & & & & & & & & & & & & & & & & & 12 & 15 & 42 & 5.10 \\
\hline $\begin{array}{l}\text { Fus } G \\
\text { Lin } G \\
\text { PH G }\end{array}$ & -6 & -63 & 9 & 5.0 & & & & & & & & & 9 & -60 & 9 & 5.0 & & & & & & & & \\
\hline $6 / 8$ & & & & & $\begin{array}{l}-6 \\
-3 \\
-9\end{array}$ & $\begin{array}{l}9 \\
33 \\
21\end{array}$ & $\begin{array}{l}63 \\
48 \\
42\end{array}$ & $\begin{array}{l}7.97 \\
6.84 \\
5.99\end{array}$ & & & & & & & & & 6 & 3 & 63 & 7.38 & & & & \\
\hline
\end{tabular}

CTRL, controls; LTLE, left temporal lobe epilepsy; RTLE, right temporal lobe epilepsy; Cx, cortex; O, occipital; OT, occipito-temporal; T, temporal; ST, superior temporal; F, frontal; Med, medial; CG, cingulum; G, gyrus; Fus, fusiform; Lin, lingual; PH, parahippocampus.

comparison). These values correspond to $\mathrm{Z}$ scores of 3.72 and above in this study. A region was considered to be activated if a spatially contiguous set of voxels were all independently significant at a level of $\mathrm{p}<0.003$.

\section{RESULTS}

\section{Memory test performance}

RTLE patients retrieval performances were significantly impaired as compared with the performances of control subjects but numerically were better than those of LTLE patients. The first day, RTLE patients recalled 5.4 (2.1) words (mean $(\mathrm{SD}))$ during the immediate retrieval experimental condition whereas control subjects recalled 10.3 (4.4) words and LTLE patients only recalled 3.1 (2.5) words. The second day, during the delayed retrieval test, RTLE patients recalled 5.15 (2.3) words whereas control subjects recalled 9.9 (4.3) words and LTLE patients 2.4 (3.6) words. The difference between the performances of the LTLE and RTLE patients and the control subjects was statistically significant during both immediate $(\mathrm{p}<0.0007$ and 0.007 , respectively) and delayed $(\mathrm{p}<0.0014$ and 0.0028 , respectively) retrievals (see fig 1 ) whereas the difference between RTLE and LTLE patients was not significant.
Within the same group, we noted a very slight tendency to a decrease of the performances the second day but the difference was small and did not reach significance.

Within group comparisons of haemodynamic response Tables 1,2, and 3 show the coordinates of the areas of significant activation ( $\mathrm{p}<0.0001$, corrected for multiple comparison) during the encoding, immediate retrieval, and delayed retrieval tasks for all the subjects. Figures 2,3, and 4 show the SPM activation maps during memory tasks for the epileptic patients.

\section{Control subjects and LTLE patients}

The data concerning the healthy volunteers and the LTLE patients have already been reported. ${ }^{6}$ Briefly, control subjects and LTLE patients activated for all memory tasks a cerebral network involving the left occipito-temporo-parietal and ventrolateral frontal cortices. Right sided predominant activations were also depicted in the parahippocampal and hippocampal cortices during retrieval tasks in healthy volunteers, but not in epileptic patients. In addition, LTLE patients exhibited a dramatic new activation located in the dorsolateral frontal cortex during all memory tasks. 
Table 2 Activation foci in normal control subjects, left and right epileptic patients during the immediate retrieval tests (stereotaxic coordinates are expressed in $\mathrm{mm}$ )

\begin{tabular}{|c|c|c|c|c|c|c|c|c|c|c|c|c|c|c|c|c|c|c|c|c|c|c|c|c|}
\hline \multirow[b]{3}{*}{ Region } & \multicolumn{12}{|c|}{ Left hemisphere } & \multicolumn{12}{|c|}{ Right hemisphere } \\
\hline & \multicolumn{4}{|l|}{ CTRL } & \multicolumn{4}{|c|}{ LTLE } & \multicolumn{4}{|l|}{ RTLE } & \multicolumn{4}{|c|}{ CTRL } & \multicolumn{4}{|c|}{ LTLE } & \multicolumn{4}{|c|}{ RTLE } \\
\hline & $x$ & y & z & $\begin{array}{l}\mathrm{z} \\
\text { value }\end{array}$ & $x$ & $y$ & $\mathrm{z}$ & $\begin{array}{l}z \\
\text { value }\end{array}$ & $\mathrm{x}$ & y & z & $\begin{array}{l}\mathrm{z} \\
\text { value }\end{array}$ & $\mathrm{x}$ & y & $\mathrm{z}$ & $\begin{array}{l}z \\
\text { value }\end{array}$ & $x$ & y & $z$ & $\begin{array}{l}z \\
\text { value }\end{array}$ & $x$ & $y$ & z & $\begin{array}{l}z \\
\text { value }\end{array}$ \\
\hline $\begin{array}{l}\text { Post } C x \\
O C x\end{array}$ & $\begin{array}{l}-18 \\
-18\end{array}$ & $\begin{array}{l}-78 \\
-66\end{array}$ & $\begin{array}{l}15 \\
21\end{array}$ & $\begin{array}{l}5.28 \\
4.74\end{array}$ & -12 & -66 & 15 & 6.61 & $\begin{array}{l}-15 \\
-12\end{array}$ & $\begin{array}{l}-75 \\
-66\end{array}$ & $\begin{array}{l}18 \\
15\end{array}$ & $\begin{array}{l}6.83 \\
5.26\end{array}$ & & & & & & & & & 9 & -72 & 9 & 7.89 \\
\hline $\begin{array}{l}\text { OT Cx } \\
37 / 41 / 42\end{array}$ & $\begin{array}{l}-42 \\
-51 \\
-45\end{array}$ & $\begin{array}{l}-60 \\
-27 \\
-24\end{array}$ & $\begin{array}{l}6 \\
15 \\
3\end{array}$ & $\begin{array}{l}4.77 \\
4.83 \\
5.1\end{array}$ & & & & & & & & & & & & & & & & & & & & \\
\hline $\begin{array}{l}P C x \\
40 / 7\end{array}$ & $\begin{array}{l}-36 \\
-30\end{array}$ & $\begin{array}{l}-39 \\
-63\end{array}$ & $\begin{array}{l}48 \\
48\end{array}$ & $\begin{array}{l}5.21 \\
6.23\end{array}$ & $\begin{array}{l}-36 \\
-45\end{array}$ & $\begin{array}{l}-45 \\
-66\end{array}$ & $\begin{array}{l}42 \\
24\end{array}$ & $\begin{array}{l}5.02 \\
7.18\end{array}$ & -42 & -39 & 39 & 4.92 & & & & & & & & & & & & \\
\hline $\begin{array}{l}T C x \\
\text { ST Cx } \\
22\end{array}$ & $\begin{array}{l}-66 \\
-63 \\
-57 \\
-57 \\
-45\end{array}$ & $\begin{array}{l}-33 \\
-36 \\
-42 \\
-33 \\
-45\end{array}$ & $\begin{array}{l}9 \\
18 \\
15 \\
6 \\
18\end{array}$ & $\begin{array}{l}6.04 \\
4.66 \\
6.78 \\
6.69 \\
8.08\end{array}$ & $\begin{array}{l}-63 \\
-42\end{array}$ & $\begin{array}{l}-45 \\
-36\end{array}$ & $\begin{array}{l}21 \\
15\end{array}$ & $\begin{array}{l}5.57 \\
5.3\end{array}$ & & & & & & & & & & & & & & & & \\
\hline $\begin{array}{l}F C x \\
\text { Ventro } \\
\text { lateral Cx } \\
44 / 45\end{array}$ & $\begin{array}{l}-54 \\
-42 \\
-42 \\
-48 \\
-45 \\
-42\end{array}$ & $\begin{array}{l}24 \\
18 \\
27 \\
12 \\
21 \\
9\end{array}$ & $\begin{array}{l}6 \\
3 \\
3 \\
21 \\
18 \\
30\end{array}$ & $\begin{array}{l}4.8 \\
5.87 \\
6.62 \\
7.11 \\
6.09 \\
5.95\end{array}$ & $\begin{array}{l}-57 \\
-54 \\
-39 \\
-42 \\
-45\end{array}$ & $\begin{array}{l}15 \\
21 \\
21 \\
36 \\
51\end{array}$ & $\begin{array}{l}15 \\
3 \\
9 \\
0 \\
12\end{array}$ & $\begin{array}{l}6.78 \\
5.72 \\
5.16 \\
4.55 \\
5.07\end{array}$ & & & & & $\begin{array}{l}54 \\
39\end{array}$ & $\begin{array}{l}18 \\
27\end{array}$ & $\begin{array}{l}15 \\
9\end{array}$ & $\begin{array}{l}6.01 \\
7.72\end{array}$ & & & & & & & & \\
\hline $\begin{array}{l}\text { Dorso } \\
\text { lateral } C_{x} \\
4 / 6 / 8 / 9 / \\
10 / 46\end{array}$ & & & & & $\begin{array}{l}-57 \\
-51 \\
-48 \\
-48 \\
-39 \\
-48 \\
-42 \\
-51 \\
-45\end{array}$ & $\begin{array}{l}27 \\
30 \\
33 \\
45 \\
51 \\
18 \\
6 \\
9 \\
33\end{array}$ & $\begin{array}{l}24 \\
30 \\
21 \\
18 \\
24 \\
30 \\
30 \\
36 \\
36\end{array}$ & $\begin{array}{l}4.73 \\
5.84 \\
5.32 \\
5.03 \\
3.96 \\
6.69 \\
6.27 \\
4.78 \\
5.5\end{array}$ & $\begin{array}{l}-54 \\
-54 \\
-51 \\
-51 \\
-42 \\
-57 \\
-51 \\
-42 \\
-36\end{array}$ & $\begin{array}{l}-6 \\
3 \\
12 \\
21 \\
9 \\
18 \\
27 \\
21 \\
9\end{array}$ & $\begin{array}{l}45 \\
30 \\
39 \\
42 \\
39 \\
33 \\
27 \\
33 \\
33\end{array}$ & $\begin{array}{l}5.86 \\
5.09 \\
6.48 \\
6.45 \\
6.20 \\
4.27 \\
4.65 \\
6.07 \\
6.10\end{array}$ & & & & & $\begin{array}{l}36 \\
45 \\
48\end{array}$ & $\begin{array}{l}24 \\
21 \\
0\end{array}$ & $\begin{array}{l}36 \\
42 \\
39\end{array}$ & $\begin{array}{l}4.58 \\
6.63 \\
6.10\end{array}$ & & & & \\
\hline $\begin{array}{l}\text { Med Cx } \\
\text { CG Cx } \\
23 / 30 / 31 \\
\text { Fus G }\end{array}$ & -30 & -54 & 0 & 7.18 & $\begin{array}{l}-30 \\
-21\end{array}$ & $\begin{array}{l}-54 \\
-54\end{array}$ & $\begin{array}{l}3 \\
3\end{array}$ & $\begin{array}{l}4.87 \\
7.01\end{array}$ & & & & & & & & & $\begin{array}{l}9 \\
24\end{array}$ & $\begin{array}{l}-60 \\
-60\end{array}$ & $\begin{array}{l}6 \\
15\end{array}$ & $\begin{array}{l}7.84 \\
7.38\end{array}$ & & & & \\
\hline $\operatorname{Lin} G$ & -12 & -57 & 6 & 7.77 & & & & & $\begin{array}{l}-18 \\
-12 \\
-6\end{array}$ & $\begin{array}{l}-57 \\
-63 \\
-81\end{array}$ & $\begin{array}{l}-3 \\
6 \\
6\end{array}$ & $\begin{array}{l}3.89 \\
5.47 \\
7.89\end{array}$ & $\begin{array}{l}6 \\
15\end{array}$ & $\begin{array}{l}-60 \\
-60\end{array}$ & $\begin{array}{l}3 \\
0\end{array}$ & $\begin{array}{l}7.58 \\
4.52\end{array}$ & & & & & $\begin{array}{l}15 \\
9 \\
9 \\
3\end{array}$ & $\begin{array}{l}-57 \\
-57 \\
-63 \\
-75\end{array}$ & $\begin{array}{l}0 \\
9 \\
0 \\
3\end{array}$ & $\begin{array}{l}6.02 \\
6.18 \\
6.47 \\
7.41\end{array}$ \\
\hline PH G & $\begin{array}{l}-12 \\
-33\end{array}$ & $\begin{array}{l}-48 \\
-39\end{array}$ & $\begin{array}{l}-6 \\
-6\end{array}$ & $\begin{array}{l}7.56 \\
6.73\end{array}$ & -18 & -45 & 3 & 4.81 & & & & & $\begin{array}{l}12 \\
27 \\
21 \\
24\end{array}$ & $\begin{array}{l}-42 \\
-45 \\
-42 \\
-54\end{array}$ & $\begin{array}{l}3 \\
-3 \\
3 \\
3\end{array}$ & $\begin{array}{l}5.81 \\
7.72 \\
6.66 \\
7.31\end{array}$ & $\begin{array}{l}21 \\
27\end{array}$ & $\begin{array}{l}-45 \\
-54\end{array}$ & $\begin{array}{l}3 \\
3\end{array}$ & $\begin{array}{l}6.34 \\
6.49\end{array}$ & 24 & -51 & 6 & 5.41 \\
\hline$(6 / 8)$ & & & & & $\begin{array}{l}-9 \\
-3\end{array}$ & $\begin{array}{l}18 \\
6\end{array}$ & $\begin{array}{l}45 \\
66\end{array}$ & $\begin{array}{l}5.32 \\
7.57\end{array}$ & $\begin{array}{l}-6 \\
-3\end{array}$ & $\begin{array}{l}0 \\
15\end{array}$ & $\begin{array}{l}63 \\
54\end{array}$ & $\begin{array}{l}6.46 \\
6.45\end{array}$ & & & & & $\begin{array}{l}3 \\
6\end{array}$ & $\begin{array}{l}30 \\
18\end{array}$ & $\begin{array}{l}51 \\
48\end{array}$ & $\begin{array}{l}5.44 \\
6.86\end{array}$ & 9 & 9 & 51 & 4.79 \\
\hline
\end{tabular}

\section{RTLE patients (activation minus baseline)} Encoding task

As compared with both control subjects and LTLE patients, RTLE patients did not exhibit numerous significant left hemispheric activations during the encoding memory task (table 1). The only left hemispheric peak of activation was detected in the left dorsolateral frontal cortex (Brodmann areas 6 and 9): this activation was not present in control subjects and was much more extended in LTLE patients (eight peaks of activation in LTLE patients compared with only three peaks of activation in RTLE patients).

On the right side, two separate cortical regions were activated: the right parietal cortex (Brodmann areas 7 and 40) and the right anterior cingulate cortex (Brodmann area 32). The two right parietal peaks of activation were located within
$6 \mathrm{~mm}$ of two of the three peaks of activation detected during the encoding task in LTLE patients. The anterior cingulate activation was neither detected in control subjects nor in LTLE patients.

\section{Immediate retrieval}

RTLE patients patterns of activations were somewhat different to those of control subjects or LTLE patients during the immediate retrieval (table 2). Similarly to control subjects, RTLE patients exhibited significant activations in left occipital, parietal, and lingual regions, but no activation was depicted in the left superior temporal cortex or in the left ventrolateral frontal cortex. Significant peaks of activations, identical to those detected in LTLE patients, were depicted in the left dorsolateral frontal cortex and in the left medial frontal cortex 
Table 3 Activation foci in normal control subjects, left and right epileptic patients during the delayed retrieval tests (stereotaxic coordinates are expressed in $\mathrm{mm}$ )

\begin{tabular}{|c|c|c|c|c|c|c|c|c|c|c|c|c|c|c|c|c|c|c|c|c|c|c|c|c|}
\hline \multirow[b]{3}{*}{ Region } & \multicolumn{12}{|c|}{ Left hemisphere } & \multicolumn{12}{|c|}{ Right hemisphere } \\
\hline & \multicolumn{4}{|c|}{ CTRL } & \multicolumn{4}{|l|}{ LTLE } & \multicolumn{4}{|l|}{ RTLE } & \multicolumn{4}{|c|}{ CTRL } & \multicolumn{4}{|c|}{ LTLE } & \multicolumn{4}{|c|}{ RTLE } \\
\hline & $\mathrm{x}$ & y & z & $\begin{array}{l}\mathrm{z} \\
\text { value }\end{array}$ & $x$ & $y$ & z & $\begin{array}{l}\mathrm{z} \\
\text { value }\end{array}$ & $\mathrm{x}$ & y & z & $\begin{array}{l}z \\
\text { value }\end{array}$ & $x$ & $y$ & z & $\begin{array}{l}\mathrm{z} \\
\text { value }\end{array}$ & $\mathrm{x}$ & y & z & $\begin{array}{l}z \\
\text { value }\end{array}$ & $\mathrm{x}$ & y & z & $\begin{array}{l}\mathrm{z} \\
\text { value }\end{array}$ \\
\hline $\begin{array}{l}\text { Post Cx } \\
\text { O Cx }\end{array}$ & -18 & -78 & 18 & 6.58 & & & & & $\begin{array}{l}-15 \\
-9 \\
-6 \\
-6\end{array}$ & $\begin{array}{l}-75 \\
-78 \\
-81 \\
-69\end{array}$ & $\begin{array}{l}12 \\
6 \\
24 \\
12\end{array}$ & $\begin{array}{l}6.97 \\
7.30 \\
4.80 \\
5.22\end{array}$ & & & & & & & & & $\begin{array}{l}15 \\
12\end{array}$ & $\begin{array}{l}-66 \\
-75\end{array}$ & $\begin{array}{l}15 \\
12\end{array}$ & $\begin{array}{l}6.46 \\
7.52\end{array}$ \\
\hline $\begin{array}{l}P C x \\
40 / 7\end{array}$ & $\begin{array}{l}-51 \\
-42 \\
-39 \\
-33 \\
-33 \\
-30 \\
-30\end{array}$ & $\begin{array}{l}-36 \\
-36 \\
-36 \\
-51 \\
-60 \\
-33 \\
-45\end{array}$ & $\begin{array}{l}27 \\
36 \\
45 \\
48 \\
48 \\
48 \\
42\end{array}$ & $\begin{array}{l}4.29 \\
4.40 \\
6.24 \\
7.34 \\
7.61 \\
5.03 \\
6.68\end{array}$ & & & & & & & & & $\begin{array}{l}42 \\
36\end{array}$ & $\begin{array}{l}-39 \\
-39\end{array}$ & $\begin{array}{l}36 \\
45\end{array}$ & $\begin{array}{l}5.12 \\
5.98\end{array}$ & & & & & & & & \\
\hline $\begin{array}{l}T C x \\
\text { ST Cx } \\
22\end{array}$ & -57 & -33 & 9 & 5.95 & & & & & & & & & & & & & & & & & & & & \\
\hline $\begin{array}{l}F C x \\
\text { Ventro } \\
\text { lateral } C x \\
44 / 45\end{array}$ & $\begin{array}{l}-45 \\
-39\end{array}$ & $\begin{array}{l}21 \\
24\end{array}$ & $\begin{array}{l}21 \\
12\end{array}$ & $\begin{array}{l}5.31 \\
7.29\end{array}$ & & & & & $\begin{array}{l}-63 \\
-45\end{array}$ & $\begin{array}{l}6 \\
33\end{array}$ & $\begin{array}{l}24 \\
0\end{array}$ & $\begin{array}{l}6.18 \\
4.26\end{array}$ & $\begin{array}{l}51 \\
39\end{array}$ & $\begin{array}{l}24 \\
27\end{array}$ & $\begin{array}{l}6 \\
9\end{array}$ & $\begin{array}{l}5.96 \\
7.59\end{array}$ & & & & & & & & \\
\hline $\begin{array}{l}\text { Dorso } \\
\text { lateral Cx } \\
4 / 9 / 10 / \\
46\end{array}$ & & & & & $\begin{array}{l}-51 \\
-42\end{array}$ & $\begin{array}{l}18 \\
12\end{array}$ & $\begin{array}{l}36 \\
39\end{array}$ & $\begin{array}{l}5.70 \\
4.00\end{array}$ & $\begin{array}{l}-57 \\
-51 \\
-42\end{array}$ & $\begin{array}{l}-3 \\
24 \\
12\end{array}$ & $\begin{array}{l}45 \\
36 \\
36\end{array}$ & $\begin{array}{l}6.07 \\
7.12 \\
5.33\end{array}$ & & & & & & & & & & & & \\
\hline $\begin{array}{l}\text { Med Cx } \\
\text { CG Cx } \\
23 / 30 / \\
31\end{array}$ & & & & & -12 & -66 & 15 & 7.50 & -9 & -60 & 6 & 5.42 & & & & & & & & & 3 & -63 & 15 & 4.94 \\
\hline Fus $G$ & & & & & & & & & & & & & & & & & & & & & 21 & -60 & -3 & 5.13 \\
\hline PH G & -15 & -42 & 0 & 4.74 & -9 & -60 & 6 & 5.20 & -9 & -66 & 0 & 5.19 & 15 & -42 & 3 & 7.36 & 3 & -60 & 6 & 5.80 & $\begin{array}{l}12 \\
9 \\
3\end{array}$ & $\begin{array}{l}-66 \\
-60 \\
-75\end{array}$ & $\begin{array}{l}0 \\
6 \\
3\end{array}$ & $\begin{array}{l}7.15 \\
6.85 \\
7.28\end{array}$ \\
\hline $\mathrm{HC}$ & & & & & & & & & & & & & 24 & -39 & 3 & 7.12 & & & & & & & & \\
\hline $6 / 8$ & & & & & $\begin{array}{l}-3 \\
-3\end{array}$ & $\begin{array}{l}6 \\
15\end{array}$ & $\begin{array}{l}69 \\
60\end{array}$ & $\begin{array}{l}5.30 \\
7.30\end{array}$ & $\begin{array}{l}-9 \\
-6 \\
-3\end{array}$ & $\begin{array}{l}12 \\
21 \\
3\end{array}$ & $\begin{array}{l}57 \\
48 \\
63\end{array}$ & $\begin{array}{l}7.15 \\
4.46 \\
7.26\end{array}$ & & & & & & & & & 3 & 18 & 51 & 6.19 \\
\hline
\end{tabular}

(Brodmann areas 6 and 8), but these activations were not present in control subjects.

On the right side, we noted a new occipital peak activation that had not been detected in control subjects or in LTLE patients. Right parahippocampal activation was also detected, although less extended than in control subjects and LTLE patients (one peak of activation in RTLE patients compared with peaks of activation in control subjects and two peaks of activation in LTLE patients). Significant activations were also detected in the right lingual gyrus and the right medial frontal cortex (Brodmann area 6).

\section{Delayed retrieval}

Similarly to the immediate retrieval condition, RTLE patients hemispheric activations differed from those depicted in control subjects or LTLE patients during the delayed retrieval (table 3). Five cerebral regions were activated bilaterally: the occipital cortex, the lingual and fusiform gyri, the medial frontal cortex (Brodmann area 6), and the cingulate cortex. Left ventrolateral and dorsolateral frontal cortices were also significantly activated. Similarly to LTLE patients, no parahip- pocampal or hippocampal activations were detected in RTLE whereas significant bilateral parahippocampal activations and right hippocampal activation were present in control subjects.

\section{DISCUSSION}

The important finding of this study is that the neural networks sustaining verbal episodic memory are also altered in RTLE patients. We showed in this study that the right MTLE could lead to a left hemispheric dysfunction as demonstrated by: (a) the altered verbal memory performances of the RTLE patients and (b) the reduction of the functional activity within the left hemisphere as compared with control subjects. These results provide further evidence of the bilateral functional consequences of MTLE associated with well lateralised hippocampal sclerosis.

\section{Verbal memory score performances in RTLE patients}

RTLE patients memory performances were significantly lower than those of control subjects. This finding was relatively 


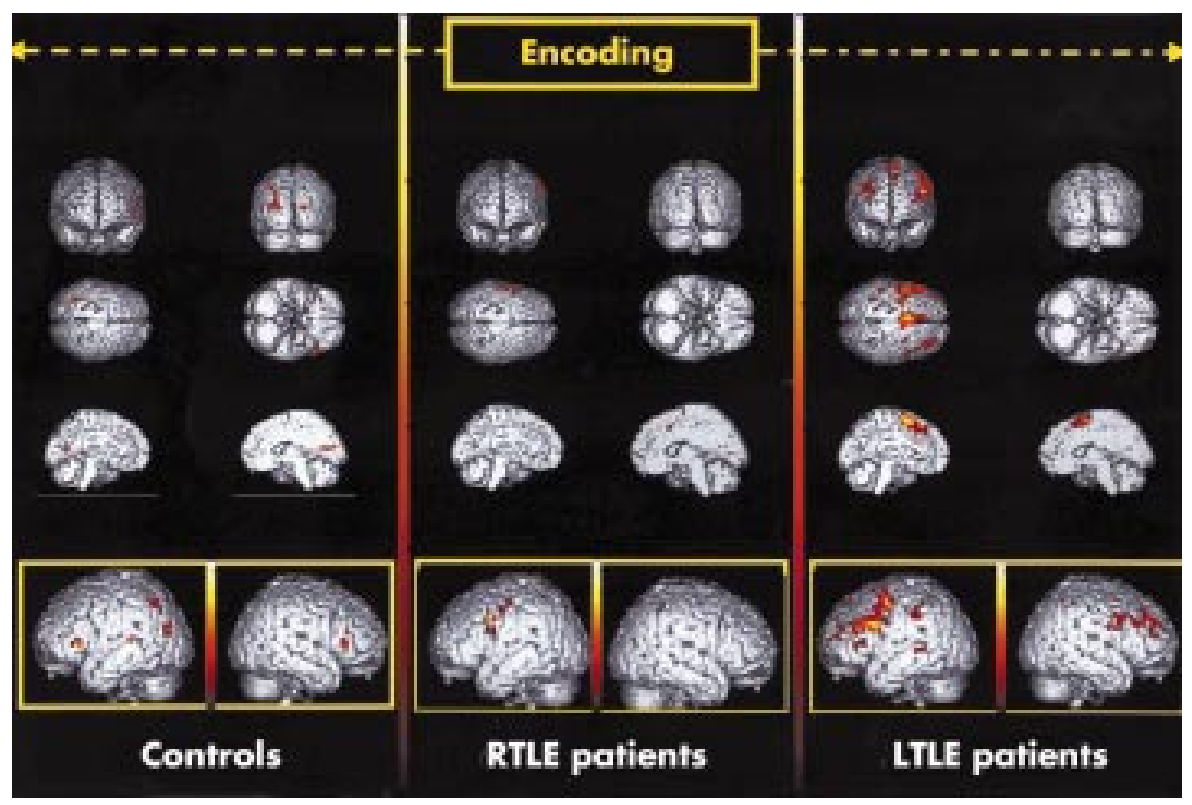

Figure 2 Statistical parametric maps of activation during the encoding tasks in control subjects and epileptic patients.

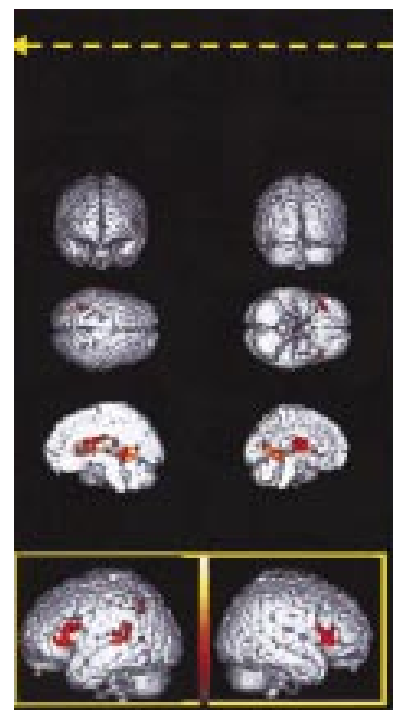

Controls

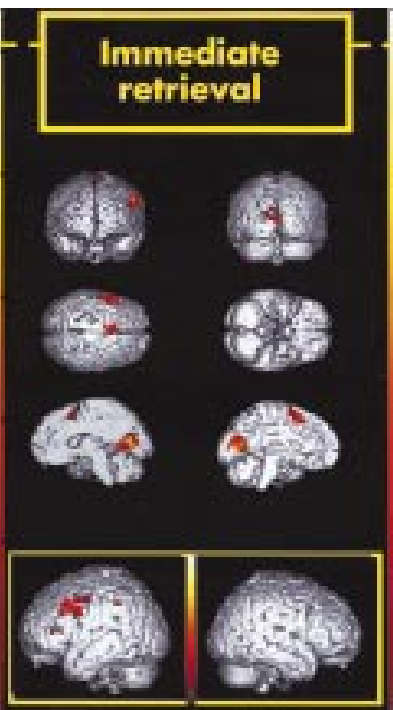

RTLE patients

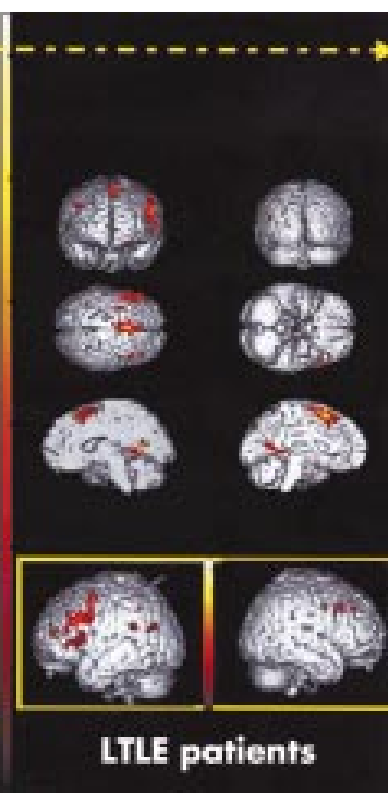

Figure 3 Statistical parametric maps of activation during the immediate retrieval task in control subjects and epileptic patients. unexpected as RTLE patients are supposed to have intact verbal memory performances. Well lateralised damage of the hippocampal structures is considered to result in material specific memory deficits, based largely upon the body of literature reporting material memory impairment after temporal lobectomy. Right anterior temporal lobectomy is known to result in spatial memory deficits, ${ }^{12}$ with sometimes some degree of discordance across postoperative studies, whereas left anterior temporal lobectomy results in verbal memory deficits. ${ }^{13}$ For many authors, verbal learning tasks seem better in distinguishing between patients with left or right hippocampal sclerosis ${ }^{13}$ with a clear correlation between neuron loss in specific areas of the hippocampus (areas CA3 and CA4) and different verbal functions in patients with left MTLE associated to left well lateralised hippocampal sclerosis. ${ }^{14}$ However, there is little information about the baseline pattern of memory ability in surgical candidates. Results of neuropsychological testing in patients with chronic MTLE have focused primarily on memory functioning, but more recent studies provided evidence of more diffuse and generalised cognitive impair- ment at the time of surgery ${ }^{15}$ with attenuated performance on measures of general intellectual functioning and neuropsychological evidence of coexisting frontal lobe compromise. ${ }^{16}$ Our study also demonstrates that bilateral memory deficits may be present in well lateralised MTLE.

\section{Memory networks in RTLE patients}

In a previous study, we showed that left hippocampal dysfunction could interfere with the normal right hippocampal function. In this study, we showed the reverse situation: a right hippocampal structural and functional failing may alter the normal function of the left hippocampus and of the overall left hemisphere. This bilateral dysfunction was supported by two findings when we compared the RTLE patients activations with the control subjects and the LTLE patients activations: (a) the global decrease in left hemisphere functional activity in terms of number of activation peaks and $\mathrm{Z}$ scores and (b) a different pattern of hemispheric activations. In the literature, a bilateral involvement has already been supported in unilateral MTLE. Some electrical and structural lines 


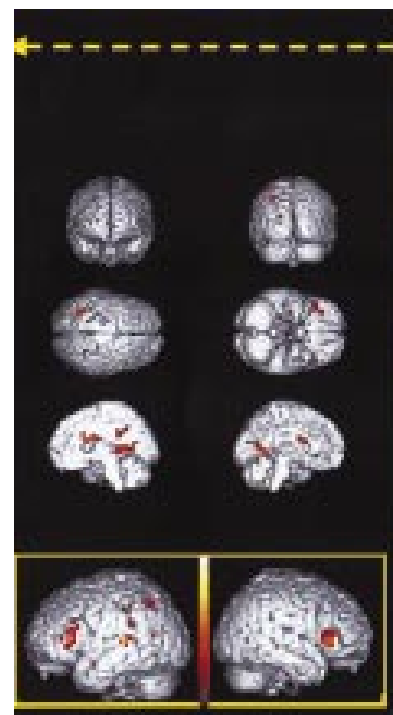

Controls

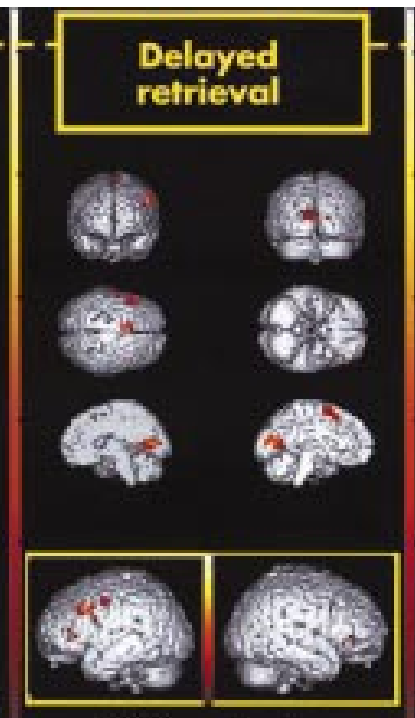

RTLE patients

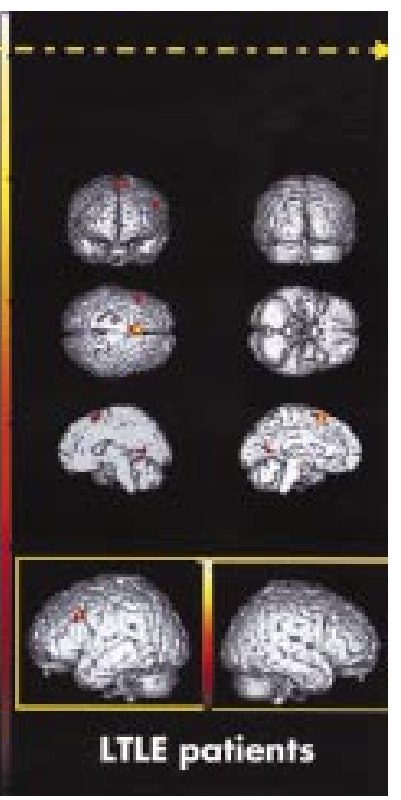

Figure 4 Statistical parametric maps of activation during the delayed retrieval task in control subjects and epileptic patients. of evidence support the idea that epileptogenic activity may affect both sides of the temporal lobe. For instance, ictal scalp and intracranial recordings may show bilateral temporal lobe onset in up to $20 \%$ of the patients ${ }^{17-19}$ whereas interictal intracranial recordings demonstrate bilateral medial temporal spikes in up to $80 \%$ of the patients. ${ }^{20}$ Similarly, the pathological basis underlying MTLE- that is, the hippocampal sclerosis is reported to be bilateral in some patients, although usually asymmetric. $^{21}{ }^{22}$ In a MRI study that investigated specifically the incidence of bilateral hippocampal atrophy in 40 patients with unilateral MTLE, Quigg $e a^{23}$ demonstrated that hippocampi contralateral to seizure onset were significantly smaller than control hippocampi, although larger than hippocampi ipsilateral to seizure onset. Furthermore, $18 \%$ of patients had bilateral atrophy, defined by volumes of each hippocampus two standard deviation lower than control means. In a similar MRI study, King et al $^{24}$ also showed that mesial temporal sclerosis could be present bilaterally in some patients as assessed by absolute hippocampal volume values and ratios measurements. In addition, numerous magnetic resonance spectroscopic imaging studies, ${ }^{25-27}$ have provided evidence of bilateral hippocampal neuronal metabolic abnormalities in patients with unilateral MTLE. Taken together, these findings suggest that MTLE induces a bilateral, dysfunctional network that may be related either to the seizure activity or to the hippocampal sclerosis. As postulated by Cendes et al, ${ }^{28}$ the seizure activity may lead to widespread neuronal functional changes and to reversible transsynaptic deafferentation of the contralateral temporal lobe. The verbal memory impairment depicted in RTLE patients could be thus attributable to the propagation of the epileptic discharges into contralateral temporal regions and/or, of course, to the anatomical consequence of the hippocampal sclerosis. The treatment - that is, the antiepileptic drugs-may also have contributed to the differences observed between the patients groups and the control subjects.

These findings suggest that, at the time of surgery, MTLE adult patients present with evidence of diffuse neuropsychological memory impairment that may be indicative of dysfunction to neural regions outside the epileptogenic hippocampus. Whether these findings are a consequence of prolonged exposure to severe and intractable epilepsy compared with the effects of the initial aetiological insult remains undetermined

In conclusion, this study shows that RTLE patients have decreased functional activity in the left MTL as compared with control subjects. This may explain why a verbal memory impairment that is usually associated with left sided MTLE can also be present in right sided MTLE, and suggests the existence of a functional bilateral hemispheric involvement in focal MTLE associated with well lateralised hippocampal sclerosis. This finding may contribute to a better understanding of the patients memory complaints and may have implications for clinical practice, such as considering earlier surgical intervention to avoid postoperative neuropsychological morbidity. However, the relative number of subjects is small in this study and we must note some caution in this regard. Larger studies are required to verify these preliminary results.

\section{Authors' affiliations}

S Dupont, Y Samson, P-F Van de Moortele, J-B Poline, D Hasboun, D Le Bihan, CEA, SHFJ, Orsay, France

S Dupont, S Samson, D Hasboun, D Le Bihan, M Baulac, Epilepsy

Unit, Clinique Neurologique Paul Castaigne, Hôpital de la

Pitié-Salpêtrière, Paris, France

Y Samson, Stroke Unit, Clinique Neurologique Paul Castaigne, Hôpital de la Pitié-Salpêtrière

S Dupont, M Baulac, EMI INSERM Cortex and Epilepsy, Faculté de la Pitié-Salpêtrière, Paris, France

Competing interests: none declared.

\section{REFERENCES}

1 Miller LA, Munoz DG, Finmore M. Hippocampal sclerosis and human memory. Arch Neurol 1993;50:391-4.

2 Delaney RC, Rosen AJ, Mattson RH, et al. Memory function in focal epilepsy: a comparison of non surgical, unilateral temporal lobe and frontal lobe samples. Cortex 1980:116:103-17.

3 Vermeulen J, Aldenkamp AP. Cognitive side-effects of chronic antiepileptic drug treatment: a review of 25 years of research. Epilepsy Res 1995;22:65-95.

4 Hermann BP, Seidenberg M, Schoenfeld J, et al. Neuropsychologica characteristics of the syndrome of mesial temporal lobe epilepsy. Arch Neurol 1997;54:369-76

5 Spencer S. Substrates of localization-related epilepsies: biologic implications of localizing findings in humans. Epilepsia 1998;39:114-23.

6 Dupont S, Van de Moortele PF, Samson S, et al. Episodic memory in left temporal lobe epilepsy: a functional MRI study. Brain 2000;123:172232

7 Adam C, Clemenceau S, Semah F, et al. Variability of presentation in medial temporal lobe epilepsy: a study of 30 operated cases. Acta Neurol Scand 1996:94:1-11.

8 Engel JJ, Van Ness PC, Rasmussen TB, et al. Outcome with respect to epileptic seizures. In: Engel JJ, ed. Surgical treatment of the epilepsies. New York: Raven Press, 1993:609-21.

9 Ogawa S, Lee TM, Kay AR, et al. Brain magnetic resonance imaging with contrast dependent on blood oxygenation. Proc Natl Acad Sci USA 1990;87:9868-72. 
10 Jones-Gotman $M$ localizing and lateralizing the epileptogenic region. In: Engel JJ, ed. Surgical treatment of the epilepsies. New York: Raven Press, 1993:245-61

11 Friston KJ, Holmes AP, Worsley KJ, et al. Statistical parametric mapping in functional imaging: A general linear approach. Human Brain Mapping 1995;2:189-210.

12 Abrahams S, Pickering A, Polkey CE, et al. Spatial memory deficits in patients with unilateral damage to the right hippocampal formation. Neuropsychologia 1997;35:11-24.

13 Sass KJ, Westerveld M, Buchanan CP, et al. Degree of hippocampal neuron loss determines severity of verbal memory decrease after left anteromesiotemporal lobectomy. Epilepsia 1994;35: 1 179-86.

14 Sass KJ, Sass A, Westerveld M, et al. Specificity in the correlation of verbal memory and hippocampal neuron loss: dissociation of memory, language, and verbal intellectual ability. J Clin Exp Neuropsychol 1992; 14:662-72.

15 Hermann BP, Seidenberg M, Wendt G, et al. Neuropsychology and epilepsy surgery: optimizing the timing of surgery, minimizing cognitive morbidity, and maximizing functional status. In: Schmidt D, Schacter SC, eds. Epilepsy problem solving in clinical practice. London: Dunitz M 2000:279-90

16 Corcoran R, Upton D. A role for the hippocampus in card sorting? Cortex 1993;29:293-304.

17 Williamson PD, French JA, Thadani VM, et al. Characteristics of media temporal lobe epilepsy: II. interictal and ictal scalp electroencephalography, neuropsychological testing, neuroimaging, surgical results, and pathology. Ann Neurol 1993;34:781-7.

18 Risinger MW, Engel JJ, Van Ness PC, et al. Ictal localization of temporal lobe seizures with scalp/sphenoidal recordings. Neurology 1989;89: 1258-93.
19 Spencer SS, Guimaraes P, Shewmon A. Intracranial electrodes. In: Engel JJ, Pendley TA, eds. Epilepsy: a comprehensive textbook. New York: Lppincott-Raven, 1998:1719-48.

20 King D, Spencer SS. Invasive EEG in mesial temporal lobe epilepsy. J Clin Neurophysiol 1995;12:32-45

21 Babb TL, Brown WJ. Pathological findings in epilepsy. In: Engel JJ, ed. Surgical treatment of the epilepsies. New York: Raven Press, 1987:511-40.

22 Margerison JH, Corsellis JA. Epilepsy and the temporal lobes. Brain 1966;89:499-531

23 Quigg $M$, Bertram EH, Jackson T, et al. Volumetric magnetic resonance imaging evidence of bilateral hippocampal atrophy in mesial temporal lobe epilepsy. Epilepsia 1997;38:588-94.

24 King D, Spencer SS, McCarthy G, et al. Bilateral hippocampal atrophy in medial temporal lobe epilepsy. Epilepsia 1995;36:905-10.

25 Cendes F, Andermann F, Preul MC, et al. Lateralization of temporal lobe epilepsy based on regional metabolic abnormalities in proton magnetic resonance spectroscopic images. Ann Neurol 1994;35:211-16.

26 Woermann FG, Barker GJ, Birnie KD, et al. Regional changes in hippocampal T2 relaxation and volume: a quantitative magnetic resonance imaging study of hippocampal sclerosis. J Neurol Neurosurg Psychiatry 1998;65:656-64

27 Woermann FG, McLean MA, Bartlett PA, et al. Short echo time single-voxel $1 \mathrm{H}$ magnetic resonance spectroscopy in magnetic resonance imaging-negative temporal lobe epilepsy: different biochemical profile compared with hippocampal sclerosis. Ann Neurol 1999;45:369-76.

28 Cendes F, Andermann F, Dubeau F, et al. Normalization of neuronal metabolic dysfunction after surgery for temporal lobe epilepsy Evidence from proton MR spectroscopic imaging. Neurology 1997;49:1525-33.

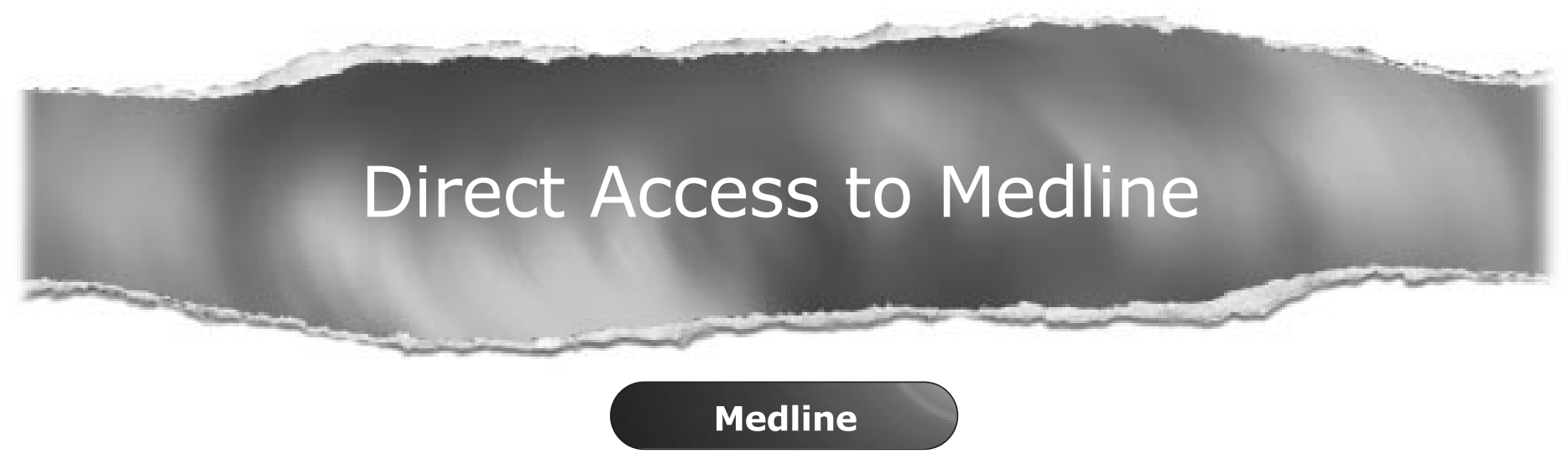

Link to Medline from the homepage and get straight into the National Library of Medicine's premier bibliographic database. Medline allows you to search across 9 million records of bibliographic citations and author abstracts from approximately 3,900 current biomedical journals.

\section{www.jnnp.com}

